\title{
STATE OF THE ART OF THE VIRTUAL REALITY APPLIED TO DESING AND MANUFACTURING PROCESSES
}

\author{
Jimeno, A.; Puerta, A. \\ Department of Computer Technology and Computation. Alicante University
}

\begin{abstract}
The idea that technology can transfer a person to a different environment without any physical movement and create the illusion of interaction with the artificial environment is not new. Scientists and engineers have been dedicating their efforts to its progressive development over the last fifty years. However, most of the technological advances have been made in the last ten years, undoubtedly thanks to improvements in computer efficiency and the miniaturization of sensorization devices. Nowadays, Virtual Reality is successfully applied in different fields, such as telemedicine, robotics or cinematography. Following on from this success, the question arises of whether we are ready to apply it to industrial design and manufacturing processes. The lack of recent reviews on this technology applied to CAD/CAM, together with its rapid evolution over the last decade, have been the primary motivations for carrying out this study.
\end{abstract}

Keywords. Virtual Reality, Virtual Prototyping, Virtual Environments.

\section{INTRODUCTION}

The Virtual Reality concept (hereinafter VR) is applied to a wide number of technologies and because of the ambiguous nature of this term in literature, it has not been fully accepted by the scientist community, [1, 2]. A definition that has been widely welcomed is the one suggested by Shuterland in his well-known The Ultimate display [3]:

A system that can display information to all senses of the user with an equal or bigger resolution than the one that can be achieved in a natural way so that the user cannot say that the artificial world is not real.

For a person to feel him or herself involved in an artificial world, it is necessary to manipulate the information perceived by his or her senses, with the perceived environment being the so-called Virtual Environment (VE), designed and created by computer and not the real physical environment. In the field of cinematography or television, VR techniques are applied to make the spectator believe in the reality of an artificial world, although fictitious actors can only interact in this new world. On the contrary, in industrial design processes, or likewise in other processes in telemedicine or robotics, the capacity to interact in the VE is more important than the visual appearance.

This article analyses the technological advances in VR that have taken place over the last decade from the point of view of CAD/CAM. 


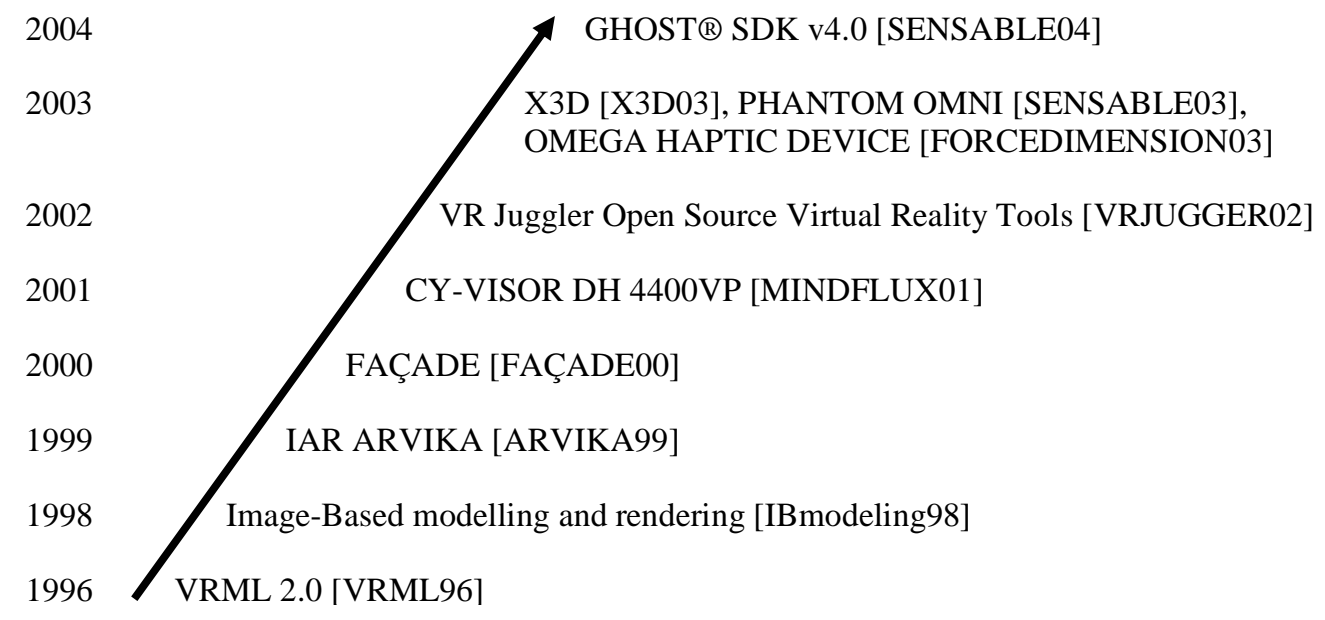

Figure 1. Some boundary works in $\mathrm{VR}^{1}$.

\section{VIRTUAL REALITY APPLIED TO CAD/CAM:}

Other technological systems, such as the television, can be included in the VR definition. However, as explained in [4], the factors that distinguish VR from any other technological system with a visual interface are presence and interaction. The sense of presence gives the user the illusion of being physically present in a $\mathrm{VE}$ and interaction the chance to modify it.

\section{Presence}

VR must give the user the sensation of being involved in the simulated environment. In [4] the explanation is given that according to the increase in the sense of presence, the sensation the user has of being part of the media environment increases and he or she seems to leave the real world where he or she is physically situated.

Some authors distinguish between immersion and presence although the two are connected. Thalmann [5] defines immersion as the sensation a VR user has of his or her VE being real. Similar to the definition of artificial intelligence by Turing, if a user is not able to determine which reality is real and which one is virtual, then the one created by computer is immersive. These factors will have to become more refined in each project so that the user can be as comfortable as possible with the VR system. Once a user-friendly VE has been achieved, the user will be able to perform actions to interact with it.

\section{Interaction}

The user present in a VE performs actions and interacts by means of hardware devices. In [6] the ways of interacting with a VE are defined as follows:

- Physical Controls: buttons, displacement bars, markers, joysticks, fliers, etc. They increase the sense of presence and are very useful for tasks requiring accuracy, although they are difficult to use while using a head-mounted display.

- Virtual Controls: any hardware device can be implemented as a virtual control to achieve great flexibility. However, they imply a loss of tactile feedback that makes interaction with virtual objects difficult.

\footnotetext{
${ }^{1}$ Web references can be found at the end of the article
} 
- Direct user interaction: following the hand, taking notes, gesture identification, eye direction, etc. They require hardware devices that perceive the user's body commands.

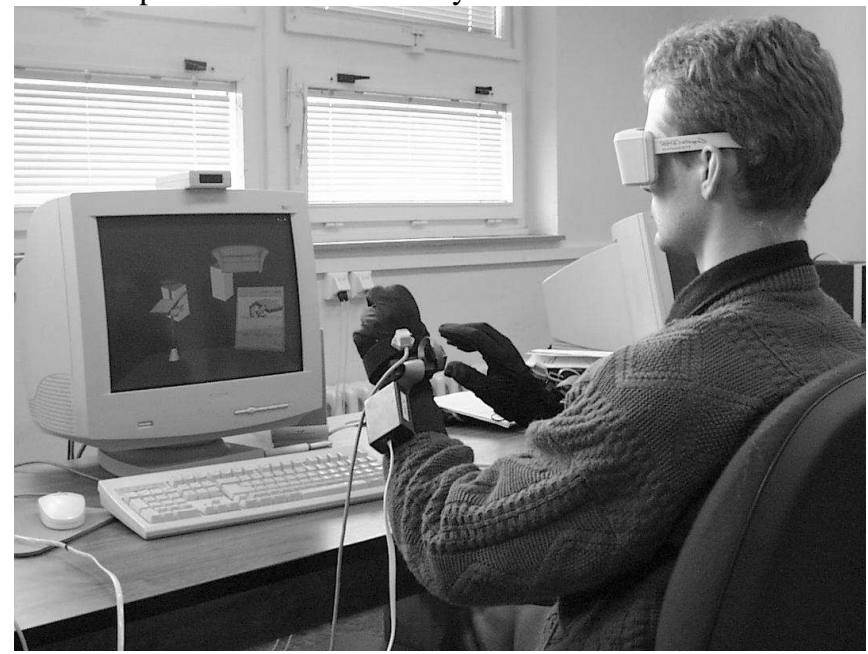

Picture. 2. Example of VR application with direct user interaction .

\section{Applications of Virtual Reality systems}

Thanks to their great ease of movement, VR systems can be employed in any application in which computer systems are used. They are particularly useful in:

- Telepresence. VR not only allows interaction with 3D worlds where the presence of the user can be a metaphor of the real one, but operations can also be executed in distant real worlds, virtual worlds generated by computer or in combinations of them both [2].

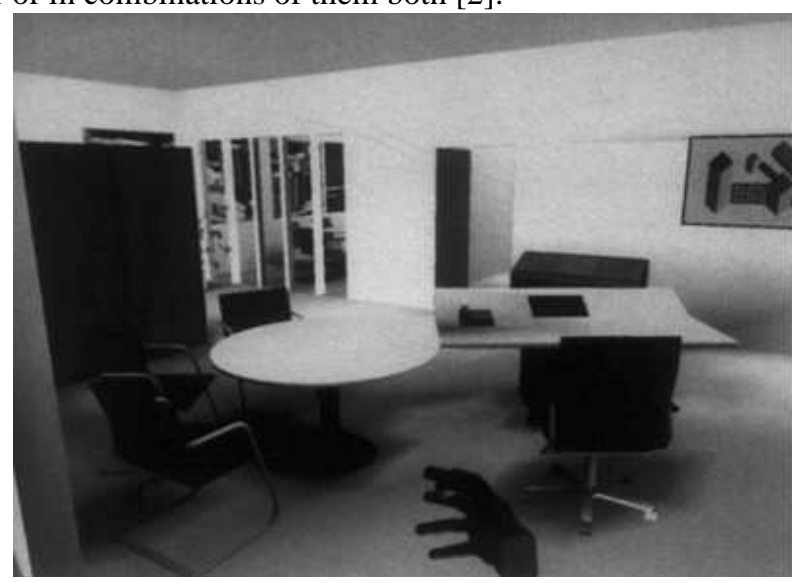

Picture. 3. The virtual representation of the user includes from avatars to the use of only one hand.

- Teleoperation. Remote controlling of a machine that operates in its environment. In the near future, robots will not possess enough intelligence to act by themselves and it will be necessary to teleoperate them. VR allows the most suitable interfaces to be created for these operations and provides feedback to the teleoperator with the robot senses [2]. 


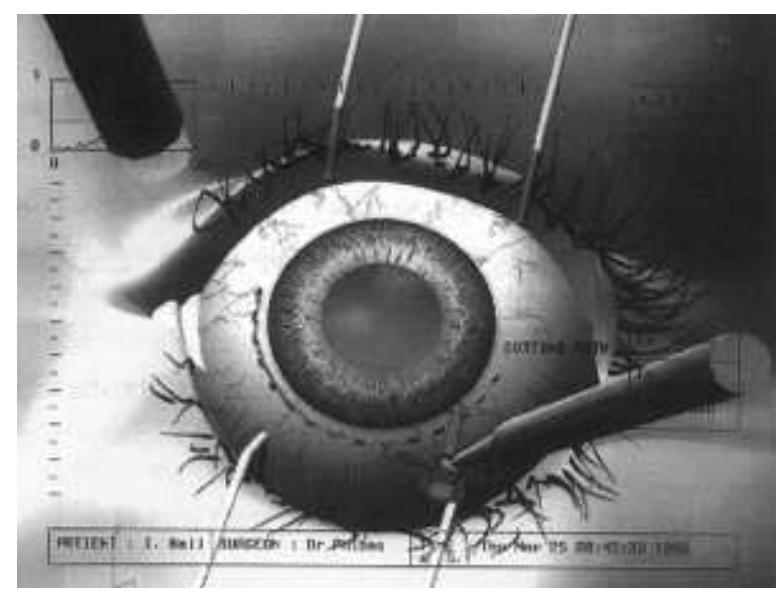

Picture 4. Medicine is one of the fields where VR is gaining more acceptance.

- Data explorer. Data modelling is an attractive use in environments where large numbers of data are generated, for example in science. It presents several levels of details and permits scientist to focus on the most relevant data [5].

- Augmented reality. VR is placed over the real world by increasing the information obtained from it. A VE that enriches the real world is represented instead of one that replaces it $[7,8]$.

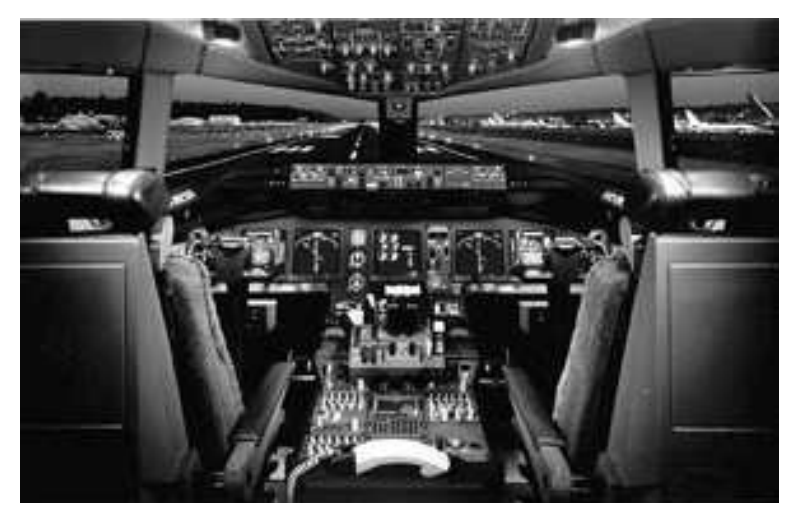

Picture 5. Simulators have greatly improved in realism by means of VR techniques.

- Virtual Sculpture. It is related to the way in which the user interacts with a 3D surface, by improving the user's tactile feedback. It allows the creation of 3D objects in a VE with the same quality as the ones created by means of physical devices or 3D environments [9].

- Virtual assembly. By means of this process, virtual prototypes are made to interact with the objects in a VE. Modern CAD systems permit the assembly of objects, but they have the disadvantages of using interfaces based on the mouse and keyboard. Virtual assembly can be performed in groups by allowing several operators to interact on the same object in cooperative environments [10]. 


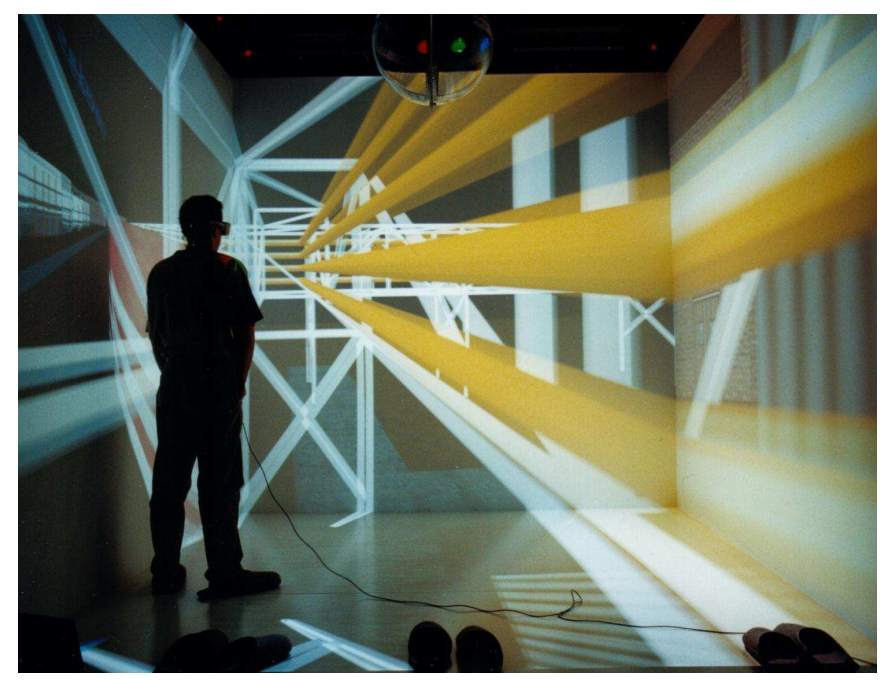

Picture 6. Example of virtual inspection in a CAVE environment.

- Virtual Prototyping. Decisions made during the design stage in large-scale projects are usually the hardest ones to make since they have the greatest effect on the final stages, prices and the product itself. Prototyping as soon as possible has become an important aim in the production cycle since it enables the team to participate in design optimization in order to eliminate weaknesses. The use of physical prototypes is more expensive, they take longer to finish and limit team work. Tactile feedback is the basis of these applications, in which the need for natural interaction and for the prototype to obtain the same features and properties as the real products, are emphasized. The prototypes highlight all the relevant features of the product that has to be investigated, evaluated and improved [11].

\section{CAD/CAM evolution}

The first CAD interactive systems were 2D drawing systems. They provided the means for the generation of pictures in the traditional way. Their main advantage was their speed. Time was saved with the use of automatic techniques for drawing symbols and for copying combinations of geometric elements.

The next improvement in development appeared early in the seventies with the introduction of the 3D wiring model. The shape of the object is represented as a set of edges in three dimensions, which provides a unified model of the object instead of several partial models, as in the case of the traditional three orthogonal view of the picture in engineering. The fact that the computer can generate pictures automatically from every point or view and in any projection chosen by the user, is an immediate advantage of the wiring model. Many CAD wiring systems allow the surfaces to be associated to the model based on edges, which permits the use of shady realistic surfaces (Non-Uniform Rational B-Spline, NURBS).

The next development was hard modelling. It combines the advantages of wiring modelling with the proper use of surfaces. As in the wiring model, the hard model includes information related to all the faces of the object together with its surface and curves. It also contains topological information which shows how these elements are interconnected in the model. A relevant advance is that most of this information is generated automatically and is checked internally by the system, which can automatically calculate the volume, mass and moments of inertia of the object.

Display modelling is used to examine both the shape and appearance. It is intended to generate not only a hard display but also a model of the product that contains semantic information from engineering [12]. These models play a crucial role in the communication of product information among the different users (marketing department, purchasers, managers, development teams, engineers, etc.). The visual appearance is also an important attractive element.

Prototypes can be classified according to the way they are generated: 
- Physical prototypes. Traditionally, material is taken from an initial block by means of a variety of processes. The result of the process is a physical prototype in wood, clay, foam or metal although it does not necessarily posses the same properties as the finished product.

- Virtual prototypes. The VP or computational prototype is generally understood to be the construction of product models by computer, frequently in a VE. This makes its assessment in a simulated functional context easier, without the need to manufacture the product first. In this way, the VP can be regarded as another way of modelling. Depending on the kind of prototype achieved, it can be divided into two groups:

- Rapid: the basis of Rapid Prototyping (RP) is the initial creation of a model by computer in three dimensions, which will become a file in digital format known as STL. Later, a computer program analyses the STL file to control the RP machine that will produce a physical prototype in a modelled material.

- Slow: this kind of prototyping is called slow, unlike the previous method, although the generic term Virtual Prototyping is usually used. The result of the process is at the same time a virtual prototype, which will be able to be explored, checked, modified and lastly used to generate the final product directly .

The reason why in [11] the VR is considered to be very useful in CAD processes is due to the disadvantages of 3D CAD devices, which are too limited to permit the interactive examination and direct manipulation of the model. With these systems, time and imagination are wasted on isolating design problems. What is more important, the lack of support for teamwork makes it very difficult for people geographically apart to share information.

In [13] the benefits of the VR systems on 3D engineering commercial devices to create digital prototypes are explained: the direct and intuitive manipulation of the virtual prototype on behalf of the user.

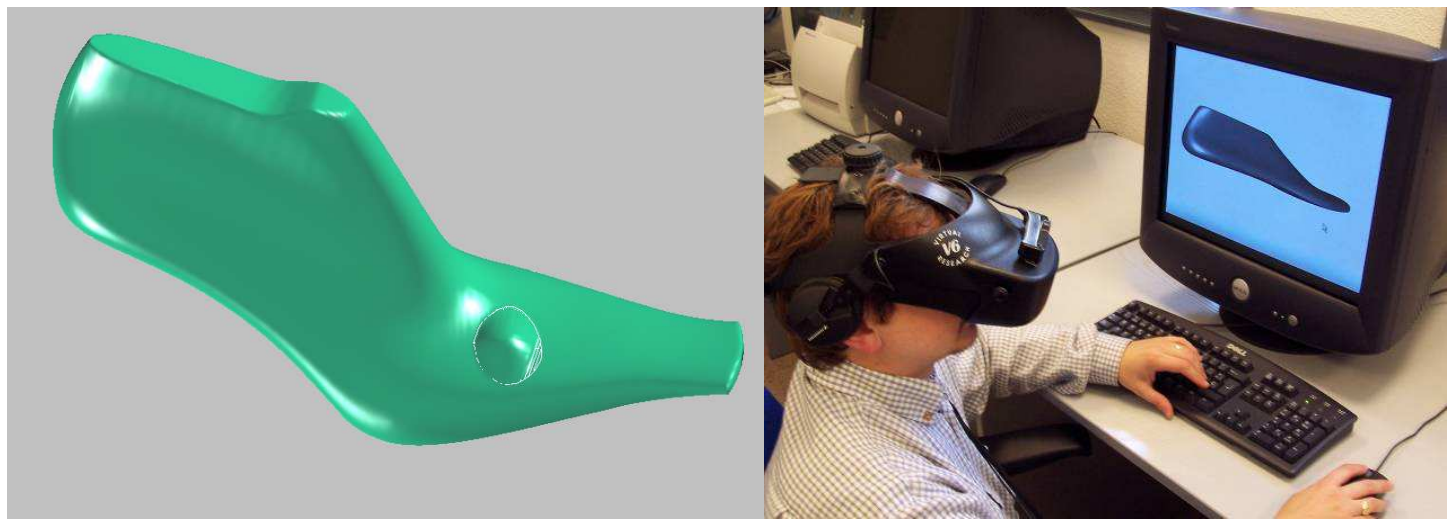

Picture. 7. Virtual devices improve the quality of the designs in CAD devices.

\subsection{Prototype generation using Virtual Reality}

The production process can be divided into three stages: design, engineering manufacturing and production. The first step of the design stage is the desired manufacture process detailed specification. This specification is the beginning of the present production process.

The design starts with a creative process in which vague concepts are manipulated until a global design appears. According to [4], the conceptual design goes through a large number of prototypes in which different aspects such as the use, manufacturing or maintenance are assessed, and changes in the design are made in each interaction.

A key aspect of the design automation is the possibility of using the design information as a basis for Computer-Aided Manufacturing (CAM) processes. CAM systems are used for automating the production 
process including both scheduling and process, controlling machines and devices. To make up CAD and CAM systems, it must be possible to use the database or generated design model in the production process.

In [14] a model is defined as an abstraction or representation of a real thing that can have many different shapes. Traditionally, the result of the computer design process is the specification of the product to be manufactured (represented in three views created by hand) and documentation. The use of CAD systems allows the computer to create the picture but other geometric description of the most sophisticated products can also be generated. CAD systems are able to generate different types of models.

The complexity of the realization cycle for mechanical products is suitable for the generation of various models of the same product for their subsequent use in different activities within the whole process. These models can be basic in the first design stages but good enough to provide responses. The design output must include a detailed geometric description of the product.

\subsubsection{Rapid Prototyping}

The manufacturing process using RP adds cuttings to the original model generated from the STL file. Layer after layer is cut until a physical model very similar to the original design is obtained.

According to [15], RP helps to produce prototypes with relative speed for their visual examination, ergonomic assessment, and shape analysis, such as main patterns for production appliances and for accelerating the complete process of the development of a product.

In the field of industry, the VR enables displays to be examined without the need for a physical prototype, at least in the first stages of the process. The classic design usually ends up as a real prototype that will be able to perform a request analysis, redesign, etc. together with the customer. However, VP enables the examination be realistic enough so that the physical prototype can be delayed. In [11] the fact that the physical prototype is not removed is highlighted although it does not need to be used so early in the design cycle.

Despite their advantages, the present RP systems are far from being ideal:

- The process has many problems that affect prototype accuracy and quality. The calculation of a RP process is affected by too many parameters and choosing a suitable combination of them is not easy. The making of the prototype depends on the quality of its request: accuracy, time of creation, resistance and efficiency of manufacture.

- Producing high-quality prototypes requires a lot of experience. The process is based on test and error, and has a very high financial and time cost.

- Depending on the RP process used, several secondary tasks, such as the extraction of remaining material, may be required after processing to finish the RP model. The accuracy of these models can be affected by the operations after processing, especially if they are done by hand.

- Once the physical prototype has been constructed, it is difficult or impossible to modify it. If problems are found with regard to the shape, for example, a new prototype will have to be created from another block of material. Although physical prototypes considerably reduce design errors, members of different development teams cannot share their knowledge; they have to work sequentially on the prototype with delays and often after frequent repetitions. 


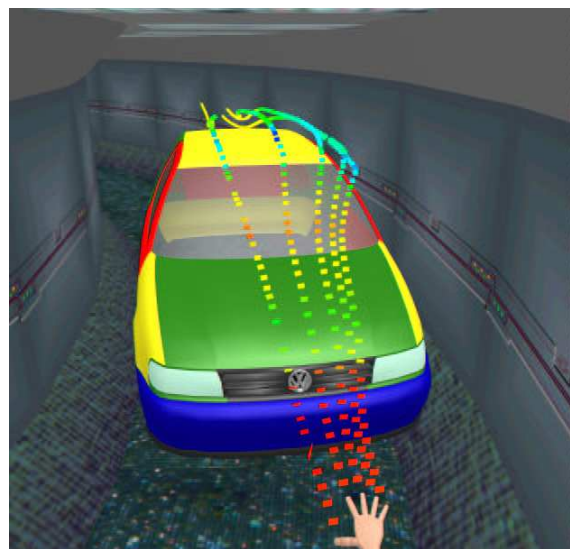

Picture. 8. In a VE, performance trials that would require expensive real instalments are possible.

\subsubsection{Virtual Prototyping}

VP can reduce the disadvantages of RP. It makes use of a digital model called a virtual prototype (instead of a physical prototype) to test and assess the specific features of a product or a manufacturing process in a computational environment.

Virtual Prototyping (VP) is the VR application in CAD/CAM processes. These applications comprise the sculpture and virtual mechanization for developing products using VR in order to obtain models which can be easily manufactured. The VP, as the VR, has been defined in different ways. In [16] it is stated:

"A virtual prototype or digital moke-up is a computer simulation of a physical product that can be presented, analyzed and tested for aspects related to the life of the product such as the design, engineering, manufacturing, service and recycling, the same as with a physical model. The construction and testing of a virtual prototype is called Virtual Prototyping (VP)."

Simulated systems are beginnning to be used in industry; however physical prototypes are still more frequently used due to their spatial presence. Physical prototypes are especially important in conceptual design and product presentation: they can be touched, handled and manipulated to check if they work properly. According to [11], VP is the technology that will be able to create digital models that permit a realistic, intuitive presentation and direct manipulation.

In [17] the benefits offered by the VP technology are listed:

- A substantial reduction in development time and in manufacturing costs thanks to the reduction in the need for expensive physical prototypes.

- The ability to keep virtual prototypes in continuous synchronization with the design together with the possibility of using them for documentary purposes. It also helps to establish a productive dialogue among engineers from different areas.

- The possibility to use prototypes during sessions of collaborative design among members geographically apart ; a very complicated option when using physical prototypes. 


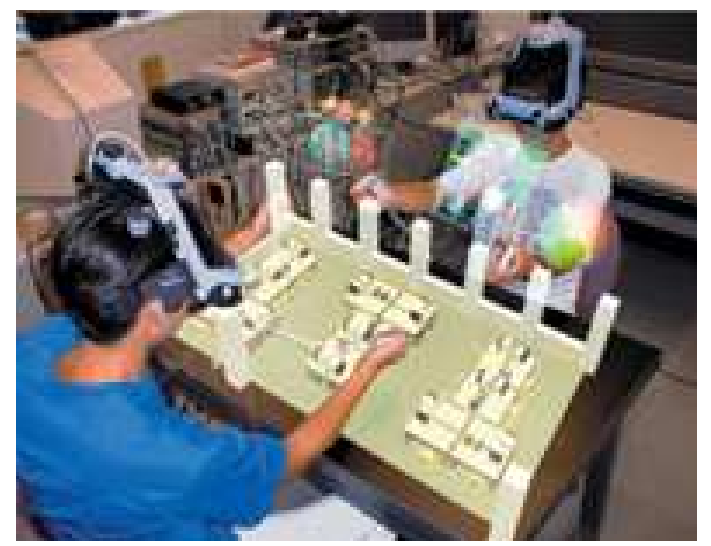

Picture. 9. Example of collaborative VE for the assembly of pieces.

- The errors resulting from the manufacture, design and planning of the product can be detected quickly, which decreases the number of physical repetitions. Once the VP is finished, the model can be used directly for its physical construction or can be sent to customers for their comments. Since digital models are the most frequently used ones in VP, the costs arising from repititions of the process to improve prototype quality are kept to a minimum.

In [18] some disadvantages of the VP systems are listed:

- A VR system prepared for VP has to handle great mathematical complexities that in general cannot be reduced using common techniques, such as texture or detail level.

- The VP simulates complex tasks involving many objects whose qualities and properties must be copied as perfectly as possible. Presence and interaction becomes more complicated.

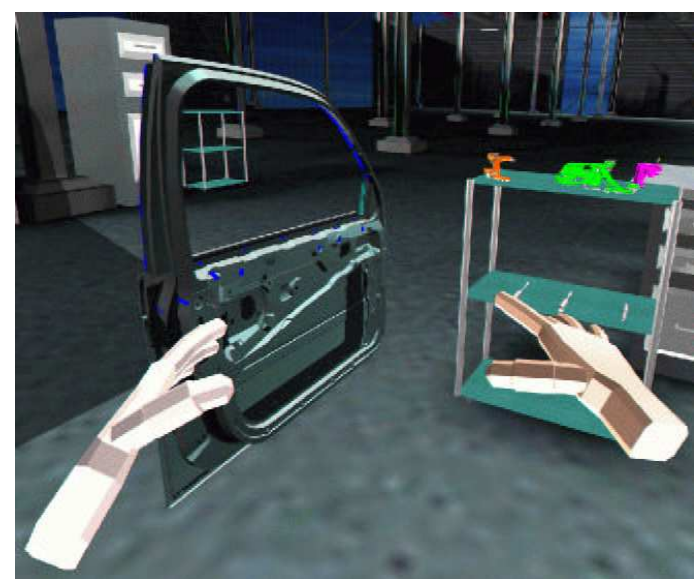

Picture. 10. Being able to analyse prototypes in a VE requires good graphics and interaction possibilities.

\subsubsection{Virtual Manufacturing}

Virtual Manufacturing (VM) involves the simulation of a product and the processes involved in its fabrication. Simulation technology enables companies to optimize key factors that directly affect the profitability of their manufactured products. These include manufacturability, final shape, residual stress 
levels, and product durability. They have a direct influence on profitability by reducing the cost of production, material usage, and warranty liabilities. In addition, virtual manufacturing also reduces the cost of tooling, eliminates the need for multiple physical prototypes, and reduces material waste.

In [29] some issues for developing a virtual turning lathe by using virtual manufacturing technology are discussed, i.e., representation of a workpiece capable of transferring error data used for machining accuracy prediction. A virtual machining and inspection system (VMIS) for ultra-precision diamond turning is also presented.

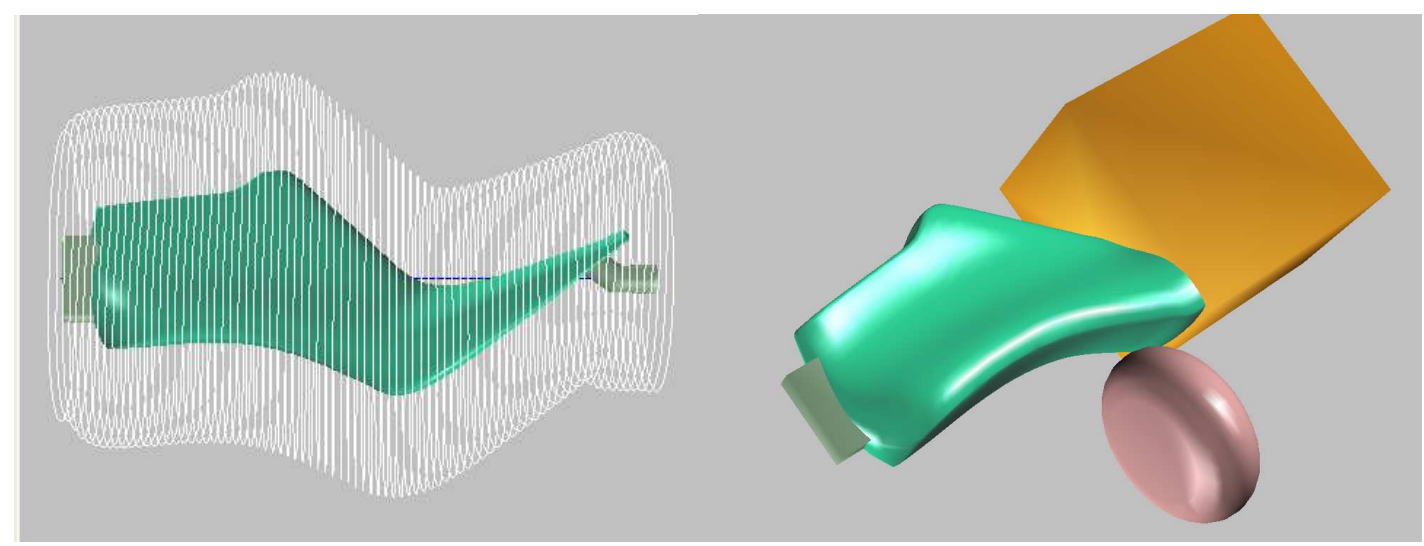

Picture 11. Virtual shoe last machining for a turning lathe.

\subsection{Disadvantages of Virtual Reality}

In [19] several aspects for improving the new VR systems appear:

- Designing in a VE. Most of the VR systems require geometrical models to be created in a CAD system before being imported to a VE where changes that cannot be exported back to the initial CAD system are made.

- Physical interaction with the design. The creation of a physical prototype is required in some stage of the design process in order to remove manufacturing risks. Unfortunately, tactile feedback in VR is in its early stages and still has a long way to go.

- Making up both real and virtual environments (augmented reality). There is a great potential without exploiting the use of real environments grown or improved with virtual technology.

- Cooperative work. The VE will have to be cooperative in order to allow several people who are physically apart to inspect or modify the prototype.

- Accuracy. Virtual prototypes will have to be created with the same accuracy and exactness as if they were real, otherwise prototypes will not be valid for study purposes. Accuracy in the interaction with objects from VE depends to a great extent on the virtual and physical devices of the user.

- Processing speed. The changes made on the virtual prototype will have to be reflected in real time, otherwise the good visual effects produced by this technology will be completely lost. According to [20], when an object is made by CAD, both accuracy and work in real time are essential.

- Purchasing and data preparation. If the data in the design are in digital format, they will have to be turned into a useful format, see UDAFS, IGES or STEP [11]. The next problem we find is that the data are usually standing for shape-free surfaces, so a tessellation has to be made to obtain the data in a polygonalized format given that the hardware is usually improved to render this kind of geometries (especially triangles). Subsequently, there will have to be a reduction in the complexity of the model obtained in order to improve the drawing speed, since maximum drawing accuracy is not as essential as the possibility of working in real time. Finally, visual information such as, material properties, colour, light, texture, etc, has to be included in the model. 
According to [21], there are side effects attached to the long-term use of VR systems. Most of them are still unknown whereas others have already been experienced by participants working in this kind of environment. These effects are known as computer simulation disease and its symptoms include: feeling sick, visual fatigue and spatial disorientation. The problem lies in the "clash of tracks" that takes place when the corporal senses receive erroneous information between reactions in real time and the visual and physical actions resulting from participation in the simulations. Computer simulation disease does not seem to have an effect on those who participate in a VR experience for only about twenty or thirty minutes, however the same is not true for those who train several hours a day in a flight simulator. The effects are also related to the problem of the kind of interfaces used, the delay attached to them and the level of immersion in which people are working within the simulated atmosphere.

Headaches and feeling sick are side effects accepted by some manufactures of devices that provide VR systems as a result of their use. In [4] different interpretations are shown about the possibility of irreversible damage.

The first clash is the one produced between the sight and the inner ear where our balance sensors are to be found. With a virtual reality device we can "see" that we fall down while our ear is still sending signs of normal conditions to our brain. This incongruity between the two senses creates unpleasant discomfort, such as sickness, although after participating in this experience over and over again, these effects are overcome or reduced. $61 \%$ of people suffer discomfort in the first session but the number decreases by half in the second session and so on. However, not everyone can adapt to VR; an estimated 5\% will always suffer discomfort and therefore VR is not recommended for this type of people.

VR is used in the treatment of patients with mental disorders such as anorexia, phobias and autism; however, it can also produce other mental illnesses. The risk seems to be more from temporary discomforts or maladjusted use. According to speculations based on certain scientific facts, virtual reality could create new neuronal connections between the visual systems and data coming from the ear. It is as if the brain learns about a new reality and is then not able to distinguish reality from virtual reality, subsequently a serious clash would be created in daily perceptions.

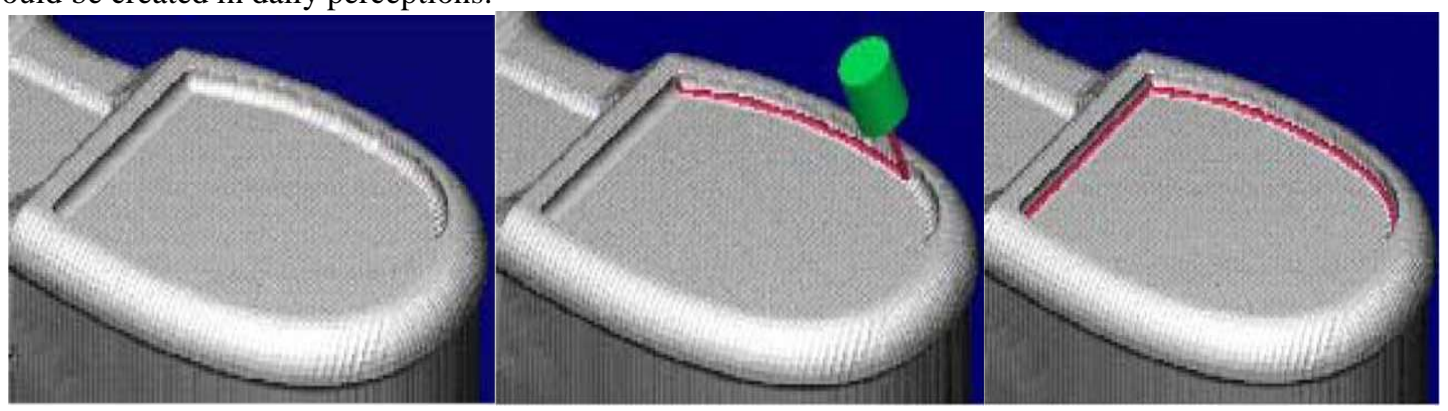

Picture. 12. Feedback devices permit great accuracy.

\subsection{Hardware devices in CAD/CAM.}

A VE will obey the "physical" laws that are programmed on it. This gives the user great flexibility with the kind of applications used in VR. Any kind of implemented application in 2D can be improved with VR. Nevertheless, the cost of developing VR systems limits its use to high profit-making applications and sectors, although there are some proposals to simplify the creation of 3D environments by means of virtual world generators or generic interfaces. 


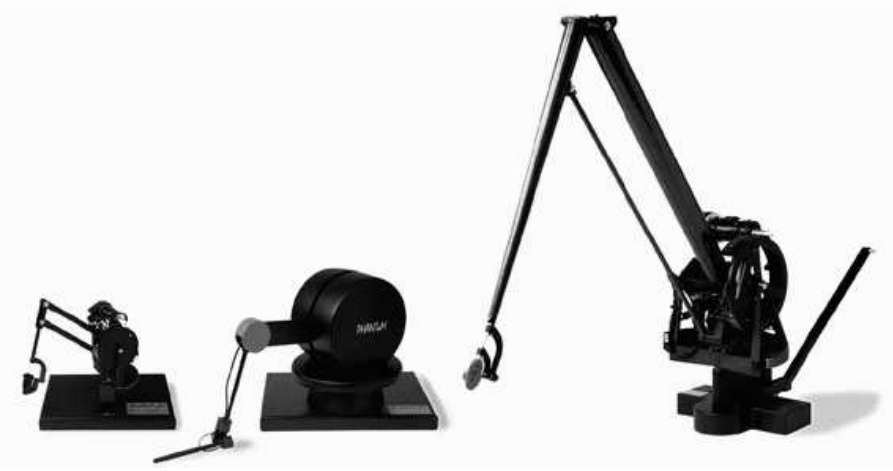

Picture. 13. One of the best ranges of products in CAD applications is Sensable.

To enter a VE, the user needs hardware or physical devices that carry him or her into it. Once in the VE by means of physical mechanisms, he or she will stimulate his or her virtual representation and will interact with his or her environment, the most essential part being the machine or computer power which all 3D devices are connected to. Without the proper power, the data sent by the physical devices will not be processed and the VE will not be modified in time.

In a 3D environment the user has to be able to interact with the 3D world in the same way as he does with the real world. The need to learn to use the system is reduced by increasing its naturalness, the sensation of reality and improving presence and interaction. The interfaces of the 3D environments must be an easy and intuitive extension of the real world.

Interaction with a VE not only requires physical devices that allow communication but also something to communicate with: the software interfaces. Virtual controls are mechanisms created in the VE and used by the user's virtual representation. These mechanisms allow the user to manage VE conditions and to carry out complex actions for physical devices.

There is a wide range of devices on the market directed above all towards visual, tactile and sound aspects, the senses most frequently used to interact both in the real and the virtual world [22]. Physical mechanisms can be classified into three large groups, depending on the type of information provided, according to $[2,5$, 23, 28]:

- Input Mechanisms: they allow data to be introduced into the VE; the human being interacts with the world that surrounds him or her through the movement of his or her body, hands and spoken demands. The most common access mechanisms are those of tracking, body movement and microphones.

- Output Mechanisms: they provide data to the user from the VE by stimulating his or her senses, sight (the most important one), hearing, and touch (feeling of contact and strength needed to interact with other objects), smell and taste. The most common output mechanisms are HMDs, BOOMs, CAVEs and amplifiers.

- Feedback mechanisms: they provide information from the VE to the user and to the VE of the position and actions made by the user. The most important ones are visual and tactile.

Picture. 14. Example of feedback mechanisms in applications in medicine.

In [24] varieties of strength and tactile power are included. The making up of strength power in a VR simulation and data about the hardness, weight and inertia of an object are provided. Tactile feedback is used to provide the user with the feeling of geometric contact with the surface of the object, smoothness, slippage and temperature. Finally, the propioceptive feedback gives the user the feeling of body position.

The strength feedback mechanisms for CAD/CAM have to take some key questions into account: the degree of freedom, the interfaces covering, maximum hold strength levels, structural rubbing and stiffness, dynamic rank, the control strip width, etc.

According to [25], with tactile interfaces engineers can touch a model to feel and modify it, by pushing, pulling and dragging its surface in a 3D natural environment. However, at present, touching is more often used to verify CAD designs than to modify them. CAD tactile geometry models are based on different displays. Most of the disfigured systems designed use traditional mechanisms. A greater importance is given 
to the ease or manipulation of the system than to interaction with objects. Traditional mechanisms have from one to three degrees of freedom and are not suitable for complicated modeling tasks, such as artistic sculpturing. To make intuitive interactions with the virtual world easier; it is advisable to find the degree of freedom of the human hand and the feeling of finger movement.

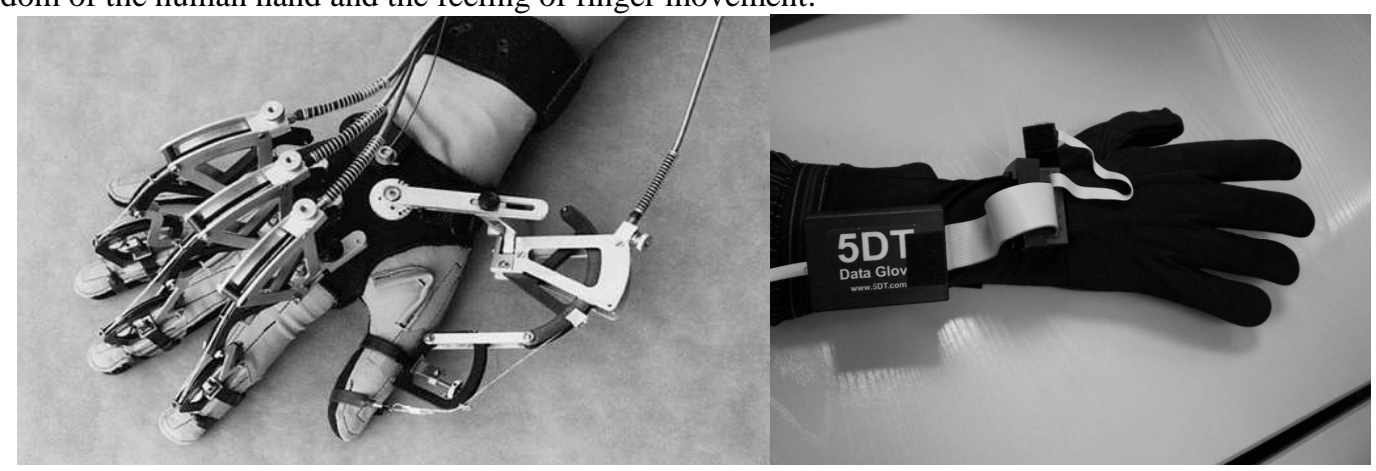

Picture. 15. VR gloves are very useful but complicated when worn during long sessions.

In [26] gloves are mentioned as the devices with the greatest natural interaction for modifying or distorting objects. This kind of device is made up of sensors that measure the movement of each finger. Commercial models include the VPL Data Glove, Virtex CberGlobe, Mattel`s PowerGlobe and Exos Dexterous Hand Master. These gloves also have sensors that measure the angles of some or all of the fingers. Some of these gloves also work with 3D trackers to find the position of the user's hand. The user manages to reach objects in the VE by means of signs of taking and pulling. By pointing the glove in the desired position, it can move or fly in that direction, always recognising the movement of the fingers.

In [24] we can find the historical evolution of commercial devices of tactile and strength feedback. In [27] we find an example of a tactile five-degree freedom tool useful in CAD/CAM systems. In [25] software technology is used to develop a tactile geometry model.

\section{CONCLUSIONS}

CAD tools do not facilitate the creative process because interfaces and functionality are unadaptable. The new generations of digital prototyping tools still cannot be adequately adapted and are based on data created in traditional CAD systems. Present devices have proved their usefulness in 2D applications but have been shown to be slow and do not fully identify with the 3D tasks to be performed.

Another problem with current devices is the feedback they offer the user both in the visual and tactile fields. All of these problems oblige the user to spend more time thinking about how to obtain what he wants in a VE compared with the time required to obtain it.

Several improvements [20] have to be made so that models created by design in a VE are of a suitably high quality for manufacturing purposes.

One of the restrictions of present VP technologies is the lack of a method for the exchange of data among different tools [12]. The integration among design, analysis and simulation tools must be improved.

User health problems, which begin after long-term use of VR systems, will have to be studied in greater depth. Although they are not determinant for the introduction of these systems, their study will help to avoid negative secondary effects.

\section{REFERENCES}

[1] Steuer, J. Defining Virtual Reality: Dimensions Determining Telepresence. Journal of Communication, 42(4):73-93, 1992. 
[2] Mazuryk T., Gervautz M. Virtual Reality History, Applications, Technology and Future. Technical Report TR-186-296-06, February 1996.

[3] Sutherland, I. E. The ultimate display. Proceedings of IFIPS Congress (New York City, NY, May 1965), vol. 2, pp. 506--508.

[4] Schumie M. J., Van der Straaten P., Krijn M., Van der Mast C. Research on Presence in VR: a Survey. Journal Cyberpsychology and Behavior Delft, 7 jan 2001.

[5] Thalmann N. M., Thalmann D. Virtual Reality Software and Technology. Encyclopedia of Computer Science and Technology, Marcel Dekker, Vol. 41, 1998.

[6] Mine M. R. Virtual Environment Interaction Techniques. Technical Report TR95-018, UNC Chapel Hill CS Dept.

[7] Azuma R. T. A Survey of Augmented Reality. Presence: Teleoperators and Virtual Environments 6, 4 (August 1997), 355 - 385. Earlier version appeared in Course Notes \#9: Developing Advanced Virtual Reality Applications, ACM SIGGRAPH (Los Angeles, CA, 6-11 August 1995), 20-1 to 20-38.

[8] Rosenblum L., Macedonia M. Developing Killer Apps for Industrial Augmented Reality. IEEE Computer Graphics and Applications, May/June 2004.

[9] Steed A. A Survey of Virtual Reality Literature. Technical report Queen Mary and Westfield College, Department of Computer Science, March 1993-623.

[10] Jung B., Hoffhenke M., Wachsmuth I. Virtual Assembly with Construction Kits. Presented at 1997 Design for Manufacturing Conference (DFM'97), September 14-17, 1997, Sacramento, CA. Appeared in Proceedings of the 1998 ASME Design for Engineering Technical Conferences (DECT-DFM '98).

[11] Dai F., Felger W., Frühauf T., Göbel M., Reiners D., Zachmann G. Virtual Prototyping Examples for Automotive Industries. Virtual Reality World '96 Proceedings, February 1996.

[12] Zorriassatine F., Wykes C., Parkin R., Gindy N. A Survey of Virtual Prototyping Techniques for Mechanical Product Development. Proceedings of the I MECH E Part B Journal of Engineering Manufacture, 1 April 2003, vol. 217, iss. 4, pp. 513-530(18).

[13] Gomes de Sa A., Zachmann G. Integrating Virtual Reality for Virtual Prototyping. Proceedings of the 1998 ASME Design Technical Conference and Computers in Engineering Conference, DETC98/CIE-5536, Atlanta, Georgia, September 13-16.

[14] Pratt M. J. Virtual Prototypes and Product Models in Mechanical Engineering. In Virtual Prototyping\&Virtual Environments and the Product Design Process (Eds J. Rix, S. Hass and J. Teixeira), 1995, pp. 113-128 (Chap- man and Hall, London).

[15] Choi S. H., Chan A. M. M. A virtual prototyping system for rapid product development. Computer-Aided Design, 36 (2004) 401-412.

[16] Wang G. G. Definition and Review of Virtual Prototyping. Journal of Computer and Information Science and Engineering, Volume 2, No. 3, September 2002 Technical Note 232-236.

[17] Gobetti E., Scateni R. y Agus M. Exploring Virtual Prototypes Using Time-Critical Multiresolution Rendering. ERCIM News No.44 - January 2001.

[18] Zachmann G. VR-Techniques for Industrial Applications. In: Fan Dai (Ed.): Virtual Reality for Industrial Applications. Springer 1998. Chapter 1, pages 13 - 38.

[19] Kalawsky R. S. Keynote Address: The Future of Virtual Reality and Prototyping. Actes du colloque scientifique international. Réalité virtuelle et prototypage, 3-4 juin 1999, Laval, 1-10.

[20] Böhm K., Hübner W. Virtual Reality: A New User Interface Paradigm For Industrial Applications. Imagina '93 Proceedings, Virtual Worlds, Conf., Monte-Carlo, Feb. 1993.

[21] Slater M., Usoh M. Body Centered Interaction in Immersive Virtual Environments. Artificial Life and Virtual Reality, N. Magnenat Thalmann, D. Thalmann, eds., John Wiley and Sons, 1994.

[22] Youngblut C., Jonhson R. E., Nash S. H., Wienclaw R. A., Will C. A. Review of Virtual Environment Interface Technology. Institute for Defence Analyses. Available at http://www.hitl.washington.edu/scivw/scivwftp/publications/IDA-pdf/

[23] Holloway R., Lastra, A. Virtual Environments: A Survey of the Technology. Eurographics '93: Notes for tutorial TN3. Barcelona, Spain. (Also republished in SIGGRAPH 1994 Course Notes, 17 (pp. A:1- A:36). New York, NY: ACM.)

[24] Burdea G. C. Haptic Feedback for Virtual Reality. Special issue on Virtual Prototyping. International Journal of Design and Innovation Research, Vol. 2, No. 1, pp. 17-29, July 2000.

[25] Liu X., Dodds G., McCartney J., Hinds B. K. Virtual Design Works-designing 3D CAD models via haptic interaction. Computer-Aided Design, In Press, Corrected Proof, Available online 3 December 2003.

[26] Wong J. P. Y., Lau R. W. H., Ma L. Virtual 3D Sculpting. Journal of Visualization and Computer Animation. John Wiley \& Sons, 11(3), pp. 155-166, July 2000.

[27] Weihang Zhu, Yuan-Shin Lee. Five-axis pencil-cut planning and virtual prototyping with 5-DOF haptic interface. Computer-Aided Design, In Press, Corrected Proof, Available online 17 March 2004.

[28] Gobbetti, E., Scateni, R. Virtual Reality: Past Present and Future. Virtual Environments in Clinical Psychology and Neuroscience. Ios Press: Amsterdam, Netherlands, 1998, pp 1-18.

[29] J.G. Li, W.B. Lee, Y.X. Yao, C.F. Cheung, S. To. Workpiece representation for virtual turning. International Journal of Advanced Manufacturing Technology, Springer-Verlag. 2005, vol 25, pp 857-866. 


\section{Web references:}

[ARVIKA99] http://www.arvika.de/www/index.htm

[FAÇADE00] http://www.debevec.org/Research/

[FORCEDIMENSION03] http://www.forcedimension.com/

[IBmodeling98] http://www.siggraph.org/s98/conference/papers/index.html

[SENSABLE03] http://www.sensable.com/

[VRJUGGER02] http://www.vrjuggler.org/

[VRML96] http://www.sdsc.edu/siggraph96vrml/

[X3D03] http://www.web3d.org/

[MINDFLUX01] http://www.mindflux.com.au/ 